Supplement of Atmos. Chem. Phys. Discuss., 15, 2577-2613, 2015

http://www.atmos-chem-phys-discuss.net/15/2577/2015/

doi:10.5194/acpd-15-2577-2015-supplement

(C) Author(s) 2015. CC Attribution 3.0 License.

(c) (i)

\title{
An ecosystem-scale perspective of the net land methanol flux: synthesis of micrometeorological flux measurements
}

\author{
G. Wohlfahrt et al.
}

Correspondence to: G. Wohlfahrt (georg.wohlfahrt@uibk.ac.at) 
Table S1. Details on setup and instrumentation for methanol flux measurements.

\begin{tabular}{|c|c|c|c|c|c|c|c|c|}
\hline & $\begin{array}{l}\text { Blodgett } \\
\text { Forest }\end{array}$ & $\begin{array}{l}\text { Missouri } \\
\text { Ozark }\end{array}$ & $\begin{array}{l}\text { Harvard } \\
\text { Forest }\end{array}$ & Vielsalm & $\begin{array}{l}\text { Oensingen } \\
\text { INT }\end{array}$ & $\begin{array}{l}\text { Oensingen } \\
\text { EXT }\end{array}$ & Neustift & $\begin{array}{l}\text { Stordalen } \\
\text { mire }\end{array}$ \\
\hline Flux method & REA & DEC & DEC & DEC & DEC & DEC & DEC & DEC \\
\hline $\begin{array}{l}\text { vDEC sampling } \\
\text { interval and dwell } \\
\text { time(s) }\end{array}$ & - & $1.2(0.1)$ & $3.0(0.2)$ & $2.0(0.2)$ & $0.7-1.3(0.2)$ & $0.7-1.3(0.2)$ & $\begin{array}{l}1.8-3.0 \quad(0.2- \\
0.5)\end{array}$ & $3.0(0.5)$ \\
\hline $\begin{array}{l}\text { Sonic anemometer } \\
\text { model }\end{array}$ & $\begin{array}{l}\text { CSAT, } \\
\text { Campbell } \\
\text { Scientific, USA }\end{array}$ & $\begin{array}{l}\text { CSAT, } \\
\text { Campbell } \\
\text { Scientific, USA }\end{array}$ & $\begin{array}{l}\text { SATI-K probe, } \\
\text { Applied } \\
\text { Technologies, } \\
\text { USA }\end{array}$ & R2,Gill UK & HS, Gill, UK & HS, Gill, UK & R3, Gill, UK & $\begin{array}{l}\text { USA-1, Metek, } \\
\text { Germany }\end{array}$ \\
\hline PTR-MS & - & $\begin{array}{l}\text { Ionicon } \\
\text { Analytik, } \\
\text { Austria }\end{array}$ & $\begin{array}{l}\text { Ionicon } \\
\text { Analytik, } \\
\text { Austria }\end{array}$ & $\begin{array}{l}\text { Ionicon } \\
\text { Analytik, } \\
\text { Austria }\end{array}$ & $\begin{array}{l}\text { Ionicon } \\
\text { Analytik, } \\
\text { Austria }\end{array}$ & $\begin{array}{l}\text { Ionicon } \\
\text { Analytik, } \\
\text { Austria }\end{array}$ & custom-built & $\begin{array}{l}\text { Ionicon } \\
\text { Analytik, } \\
\text { Austria }\end{array}$ \\
\hline $\begin{array}{l}\text { Inlet length/inner } \\
\text { diameter }(\mathrm{m})\end{array}$ & - & $40 / 0.0064$ & $50 / 0.006$ & $60 / 0.0064$ & $30 / 0.0035$ & $30 / 0.0035$ & $14 / 0.004$ & $12 / 0.008$ \\
\hline Inlet heating $(\mathrm{Y} / \mathrm{N})$ & - & $\mathrm{N}$ & $\mathrm{N}$ & $\mathrm{Y}$ & $\mathrm{N}$ & $\mathrm{N}$ & $\mathrm{Y}$ & $\mathrm{Y}$ \\
\hline Inlet flow (l/min) & - & 10 & 11.8 & 9 & 4 & 4 & 8 & 20 \\
\hline $\begin{array}{l}\text { PTR-MS drift tube } \\
\text { pressure (mbar) }\end{array}$ & - & 2.3 & 2.1 & 2.1 & 2.1 & 2.1 & $2.15-2.3$ & 2.2 \\
\hline $\begin{array}{l}\text { PTR-MS drift tube } \\
\text { voltage }(V)\end{array}$ & - & 540 & 600 & 600 & 550 & 550 & $550-600$ & 600 \\
\hline $\mathrm{E} / \mathrm{N}(\mathrm{Td})$ & - & 110 & 126 & 143 & 122 & 122 & 130 & 130 \\
\hline Key reference & $\begin{array}{l}\text { Schade and } \\
\text { Goldstein } \\
(2001)\end{array}$ & $\begin{array}{l}\text { Seco et al. (in } \\
\text { preparation) }\end{array}$ & $\begin{array}{l}\text { McKinney et } \\
\text { al. (2011) }\end{array}$ & $\begin{array}{l}\text { Laffineur et al. } \\
\text { (2012) }\end{array}$ & $\begin{array}{l}\text { Brunner et al. } \\
\text { (2007) }\end{array}$ & $\begin{array}{l}\text { Brunner et al. } \\
\text { (2007) }\end{array}$ & $\begin{array}{l}\text { Hörtnagl et al. } \\
\text { (2011) }\end{array}$ & $\begin{array}{l}\text { Holst et al. } \\
(2010)\end{array}$ \\
\hline
\end{tabular}



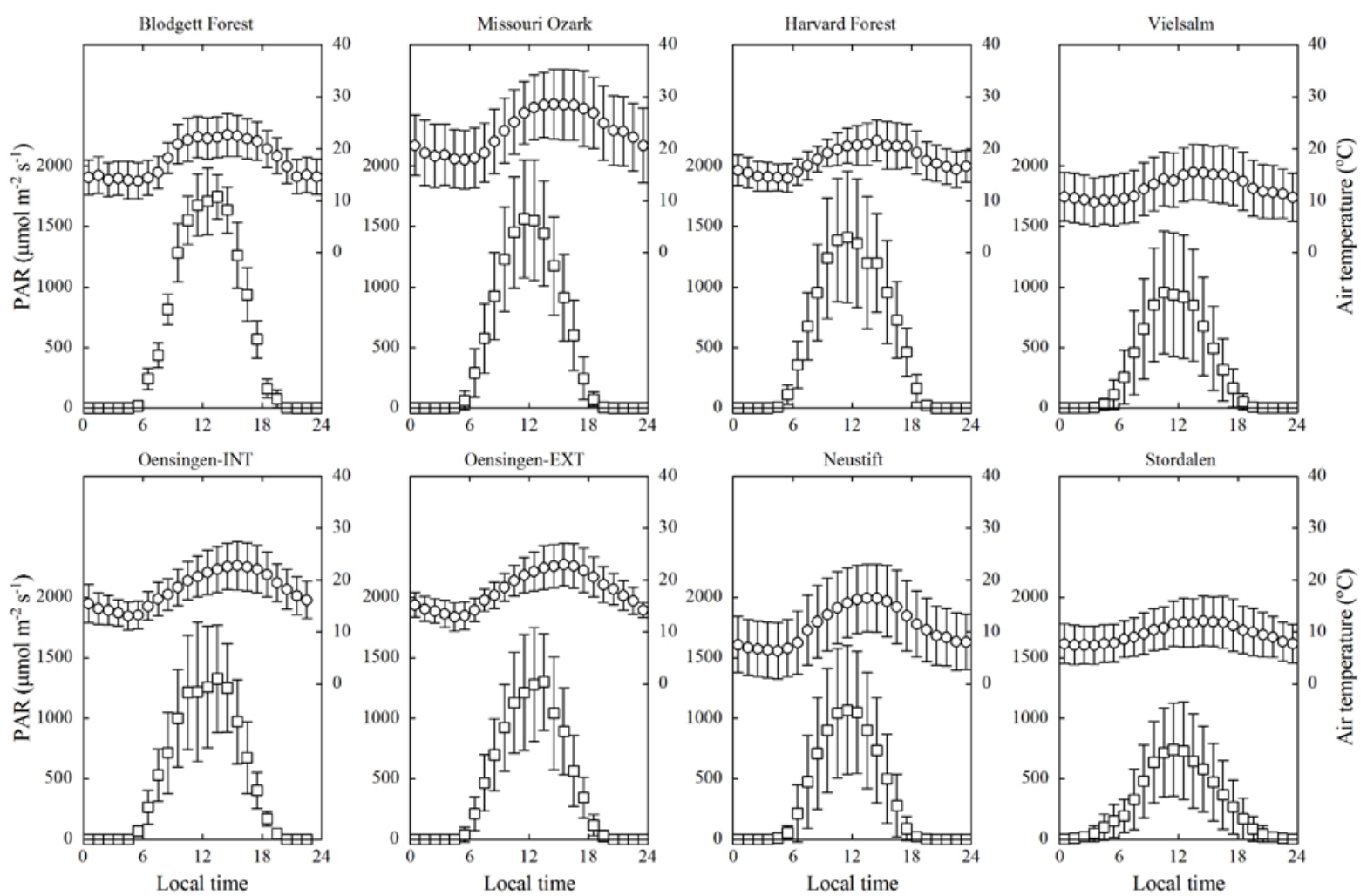

Figure S1. Hourly bin-averaged diurnal variation of photosynthetically active radiation (PAR; squares; left y-axis) and air temperature (circles; right y-axis) at the eight study sites (error bars represent \pm one standard deviation). 


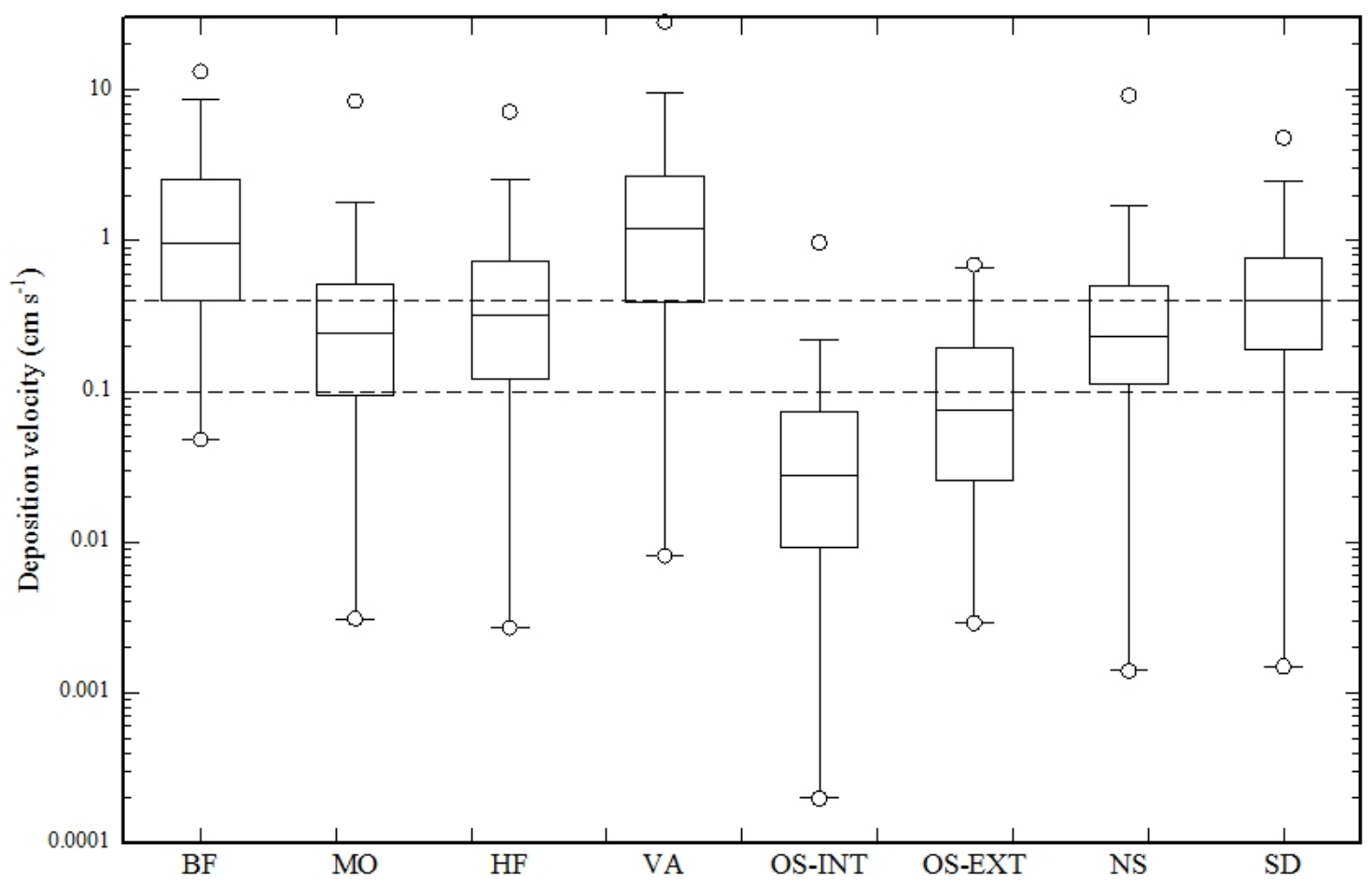

Figure S2. Box-plots of methanol deposition velocities at the eight study sites. Horizontal dashed lines indicate the range of deposition velocities $\left(0.1-0.4 \mathrm{~cm} \mathrm{~s}^{-1}\right)$ used in global budgets (see also Table 2). Box plots show minima/maxima (circles), 5\% and 95\% quartiles (whiskers), the interquartile range (box) and the median (horizontal line). 

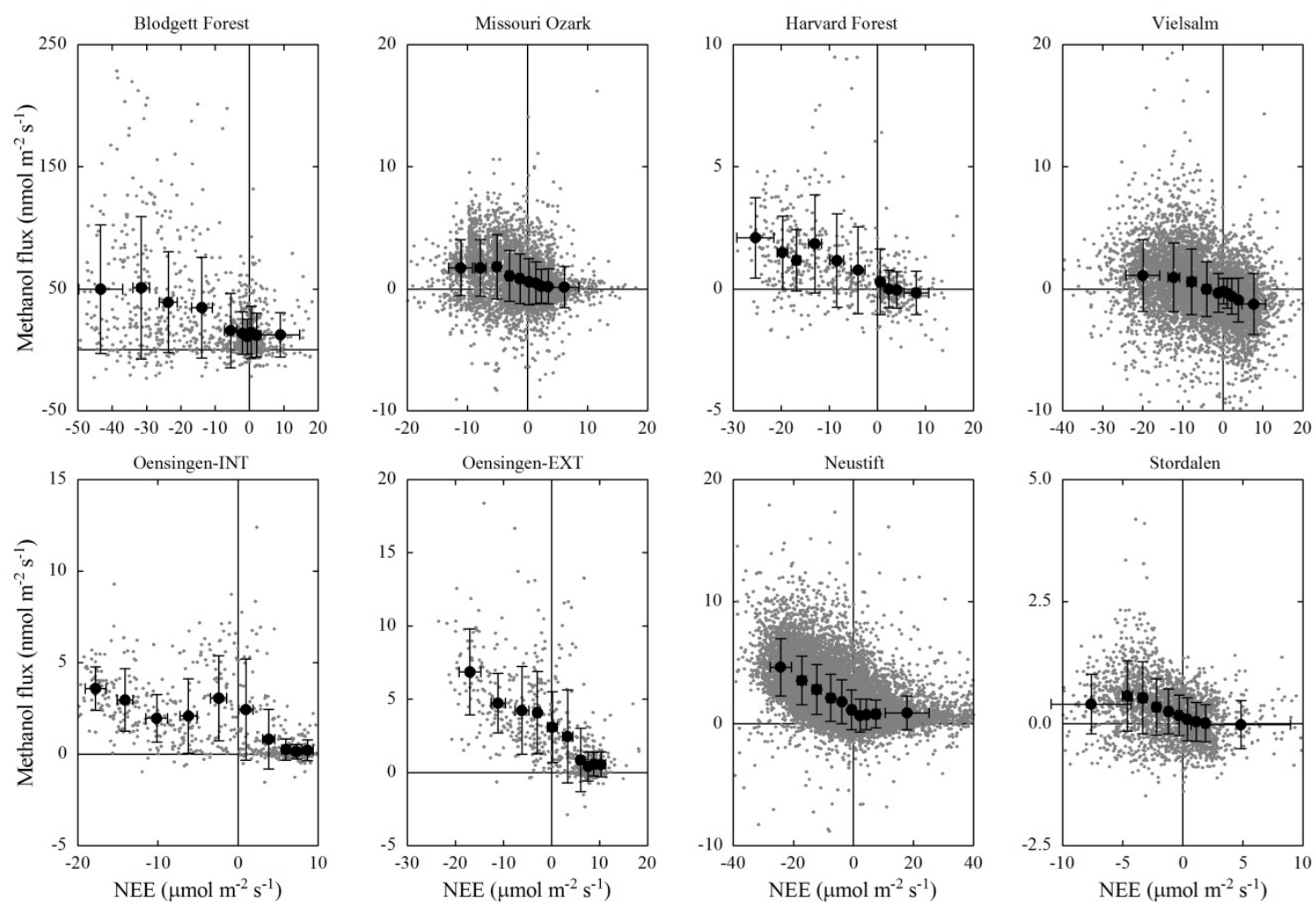

Figure S3. Relationship between the net ecosystem $\mathrm{CO}_{2}$ exchange (NEE) and methanol flux. Small grey symbols represent half-hourly flux measurements, black closed symbols 10 bin averages with equal numbers of data. Error bars refer to one standard deviation. Note different $\mathrm{x}$ - and $\mathrm{y}$-scales in different panels. 